\title{
Kinematic calculation analysis of micro injection molding machine with double-toggle clamping mechanism based on MATLAB
}

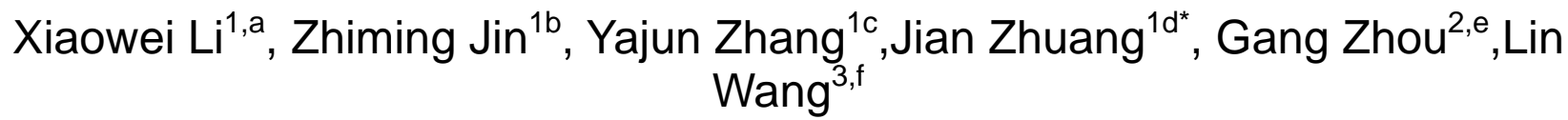 \\ ${ }^{1}$ College of Mechanical and Electrical Engineering, Beijing University of chemical technology, \\ Beijing, China, 100029 \\ ${ }^{2}$ HongKong L.K, Group, Ningbo L.K. Mechanical Co.LTD, 315806 \\ ${ }^{3}$ Patent Examination Cooperation Center of the Patent Office, SIPO, Beijing, China, 100083 \\ ali200631076@126.com, bjinzm@mail.buct.edu.cn, 'zhyj@mail.buct.edu.cn, \\ dzhuangjian@mail.buct.edu.cn, ${ }^{\mathrm{e}} \mathrm{lknblk@lknblk.com},{ }^{\mathrm{f}}$ struggle9@126.com
}

Keywords: Micros Injection Molding, Double-toggle Clamping Mechanism, kinematical Calculation

\begin{abstract}
The geometric model of micro injection molding machine with double-toggle clamping mechanism is established to analyses the kinematic and mechanical performance of double-toggle clamping mechanism. The kinematic analysis of the double-toggle clamping mechanism is used to study the effects of the double-toggle parameters for the microstructure injection molding machine. The parameters discussed in this paper include the amplification ratio, velocity ratio and the amplified force ratio for the double-toggle clamping mechanism. The optimized parameters of clamping mechanism are obtained according the calculation results of stroke ratio, velocity ratio and the amplified force ratio.
\end{abstract}

\section{Introduction}

Clamping mechanism is one of the most important execution units in the micro injection molding machines, which can provide the dimensional stability of the machine platen. The performance of clamping mechanism affects the quality of the plastic products directly. The structure of double-toggle clamping mechanism has a lot of advantages, such as high stroke ratio, high force amplified ratio, good kinematic performance, work steadily, low energy consumption and low cost $[1,2]$. The intervention and restriction each other for components should be considered when the machine structures are designed [3]. Firstly, the geometric model of the double-toggle clamping mechanism should be established, the model with the knowledge of theoretical mechanics should be analyzed. Secondly, calculation analysis should be carried out used calculation software such as MATLAB. Finally, the influences of the parameters for the design of double-toggle clamping mechanism on performance machine can be gained.

The designed toggle part in the double-toggle clamping mechanism in this paper is compared with the available injection molding machines. It is necessary to obtain the position dimensions relationship of the toggle mechanism and platen stroke, velocity and the amplified force ratio $[4,5]$. With MATLAB, the double-toggle parameters are optimized, and the influences of the physical parameters of double-toggle clamping mechanism are obtained, which provides the foundation for the design of microstructure injection molding machine. 


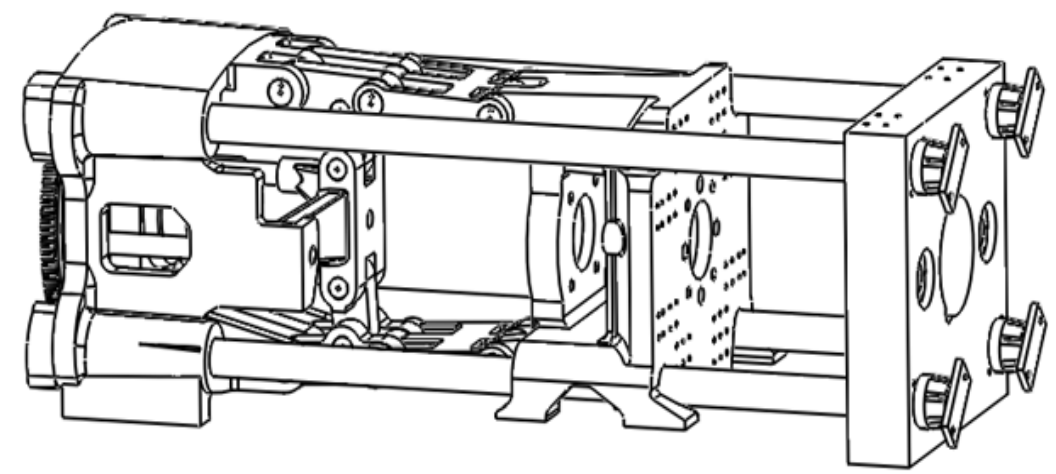

Fig.1 The double-toggle clamping mechanism of injection molding machine

\section{Performance analysis of double-toggle clamping mechanism}

The geometric model of microstructure injection molding machine is showed in Fig.2, in which the broken lines display the opening platen place, and the real lines display the closing platen place.

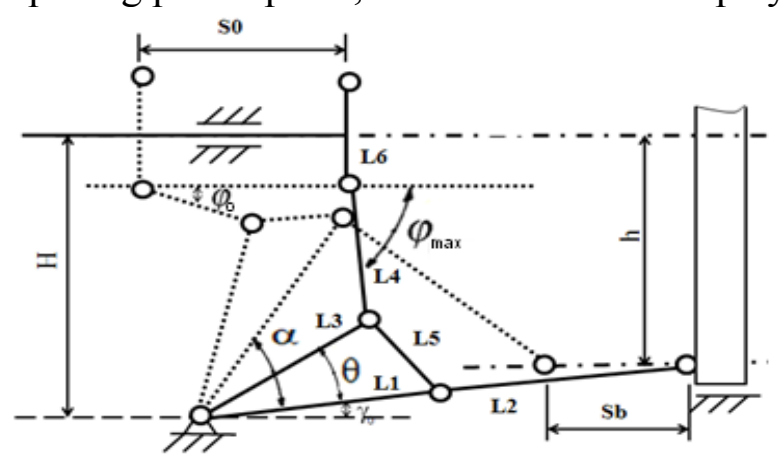

Fig.2 The geometric model of double-toggle clamping mechanism

$L_{1----l e n g t h}$ of back toggle

$L_{2}$----length of frontal rod

$L_{3}$----length of larger connecting toggle

$L_{4}$----length of nether connecting rod

$L_{5}$----length of middle connecting rod

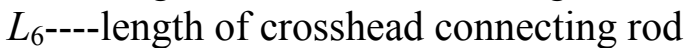

$\alpha$----toggle angle

$\varphi$----coping angle

$\beta$----angle between $\mathrm{L}_{2}$ and horizontal line
$H$----height of crosshead center

$h$----height of template center

$\gamma_{0}$----fastigiated angle

$\theta$----angle between $\mathrm{L}_{1}$ and $\mathrm{L}_{3}$

$\varphi_{0}$----maximal coping angle

$S_{0^{----}-\text {stroke of crosshead }}$

$S_{\mathrm{b}^{----}}$stroke of moving platen

$\lambda_{1}$----ratio of $\mathrm{L}_{1}$ and $\mathrm{L}_{2}$

$\lambda_{2}$----ratio of $\mathrm{L}_{3}$ and $\mathrm{L}_{4}$

\section{Stroke Ratio}

The ultimate places of opening platen and closing platen are showed in Fig.2. When the toggle stays at the ultimate place of opening platen, $\alpha=\alpha_{\max }, \psi=\psi_{0}$; when the toggle stays at the ultimate place of closing platen, $\alpha=0, \psi=\psi_{\max }[6,7]$.The stroke of crosshead $\left(S_{\mathrm{b}}\right)$, the stroke of moving platen $\left(S_{0}\right)$ and the ratio of stroke $\left(M_{\mathrm{S}}\right)$ are characterized as follows:

$$
\begin{aligned}
& S_{b}(\alpha)=L 1\left\{\frac{1}{\lambda_{1}}\left[\cos \gamma_{0}-\sqrt{1-\lambda_{1}^{2}\left(1-\cos ^{2} \alpha\right)}\right]-\cos \left(\gamma_{0}+\alpha\right)+\cos \gamma_{0}\right\} \\
& S_{0}(\alpha)=L 3\left\{\left[\cos \left(\theta+\gamma_{0}\right)-\cos \left(\theta+\gamma_{0}+\alpha\right)\right]-\frac{1}{\lambda_{2}}\left(\cos \psi_{\text {max }}-\cos \psi_{0}\right)\right\} \\
& M_{S}(\alpha)=\frac{S_{b}(\alpha)}{S_{0}(\alpha)}
\end{aligned}
$$

Velocity Ratio

The velocity of moving platen in toggle clamping mechanism represents the average velocity of the moving platen [6]. In order to obtain the kinematic performance of the mechanism, it is necessary to draw the velocity chart of the design clamping mechanism. Based on the amplification 
theory, the formulation of velocity ration of machine platen can be written as follows:

$$
M_{v}=\frac{d S_{m}}{d S_{0}}=\frac{\nu_{m}}{v_{0}}=\frac{L_{1}}{L_{3}} \sin (\alpha)\left(1+\frac{\lambda_{1} \cos \alpha}{\sqrt{1-\lambda_{1}^{2} \sin ^{2} \alpha}}\right) \frac{\cos \psi_{0}}{\sin \left(\alpha+\gamma_{0}+\psi_{0}\right)}
$$

Amplified force ratio

Toggle mechanism has the character of amplifying force, and showed by $M_{\mathrm{p}}$ which represents the relationship of force between the moving platen and the screw rod is given as follows:

$$
\begin{gathered}
V_{0} P_{0}=V_{m}(\alpha) P_{\mathrm{m}}(\alpha) \quad M_{p}(\alpha)=\frac{P_{m}(\alpha)}{P_{0}}=\frac{V_{0}}{V_{m}(\alpha)}=\frac{S_{0}}{S_{m}(\alpha)} \\
M_{p}(\alpha)=\frac{L_{3}\left\{\left[\cos \left(\theta+\gamma_{0}\right)-\cos \left(\theta+\gamma_{0}+\alpha\right)\right]-\frac{1}{\lambda_{2}}\left(\cos \psi_{\max }-\cos \psi_{0}\right)\right\}}{L_{1}\left\{\frac{1}{\lambda_{1}}\left[\cos \gamma_{0}-\sqrt{1-\lambda_{1}^{2}\left(1-\cos ^{2} \alpha\right)}\right]-\cos \left(\lambda_{0}+\alpha\right)+\cos \gamma_{0}\right\}}
\end{gathered}
$$

The relationship between $\varphi$ and $\alpha$

The function relationship between $\varphi$ and $\alpha$ can be concluded as follows:

$$
\cos \psi=\left[1-\frac{H-h-L_{3} \sqrt{1-\cos ^{2}\left(\gamma_{0}+\theta+\alpha\right)}}{L_{4}}\right]^{\frac{1}{2}}
$$

\section{Analysis and calculation based on MATLAB}

\section{Calculating parameter with MATLAB}

The double variable method is used to optimize the clamping mechanism in this paper. From the clamping mechanism geometry, the value of the parameters $\gamma_{0}, \theta, L_{6}, H, h$ can be designed. The values of the parameters are shown in Table 1.

Table 1 the values of the parameters

\begin{tabular}{|l|l|l|l|l|}
\hline$\gamma_{0}$ & $\theta$ & $L_{6}$ & $H$ & $h$ \\
\hline $3.193^{\circ}$ & $13.664^{\circ}$ & $190 \mathrm{~mm}$ & $220 \mathrm{~mm}$ & $199 \mathrm{~mm}$ \\
\hline
\end{tabular}

In this paper, $L_{1}$ and $L_{3}$ is defined as variable values, whose range of variation is $[-10,+10]$, and it can be used to calculate the parameters $L_{2}, \lambda_{1}, L_{4}, \lambda_{2}$ and $L_{5}$ by geometry relationship. The value of the parameters is shown in table 2 .

\begin{tabular}{lllllllll} 
& \multicolumn{7}{c}{ Table 2 the value of the parameters } \\
\hline Number & Parameter $(\mathrm{mm})$ & $L_{1}$ & $L_{2}$ & $\lambda_{1}$ & $L_{3}$ & $L_{4}$ & $\lambda_{2}$ & $L_{5}$ \\
\hline 1 & The actual value & 163 & 215 & 0.76 & 102 & 93 & 1.1 & 70 \\
2 & $L_{1}+5, L_{3}+5$ & 168 & 210 & 0.8 & 107 & 94 & 1.14 & 69 \\
3 & $L_{1}+10, L_{3}+10$ & 173 & 205 & 0.94 & 112 & 93.5 & 1.2 & 73.5 \\
4 & $L_{1}-5, \quad L_{3}-5$ & 158 & 220 & 0.72 & 97 & 97 & 1 & 68 \\
5 & $L_{1}-10, L_{3}-10$ & 153 & 225 & 0.68 & 92 & 98.5 & 0.93 & 67 \\
6 & $L_{1}+5, L_{3}-5$ & 168 & 210 & 0.8 & 97 & 97 & 1 & 77 \\
7 & $L_{1}-5, L_{3}+5$ & 158 & 220 & 0.72 & 107 & 94.5 & 1.13 & 60 \\
\hline
\end{tabular}

By MATLAB, the computed curve diagrams are shown as follows from Fig.3 to Fig.6.

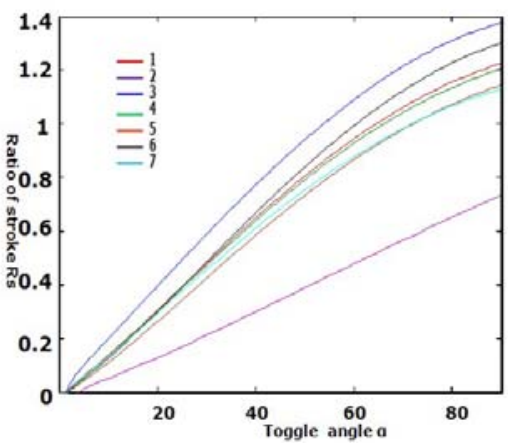

Fig.3 Relationship between journey and toggle angle of toggle mechanism

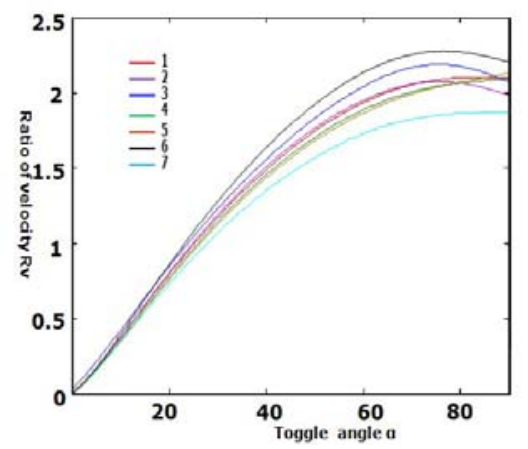

Fig.4 Relationship between velocity and toggle angle of toggle mechanism 


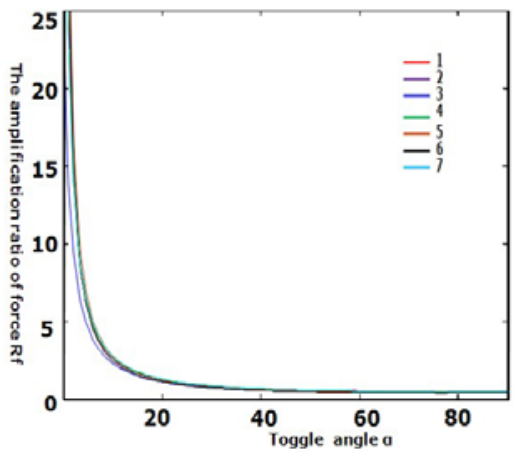

Fig.5 Relationship between force magnification and toggle angle of toggle mechanism

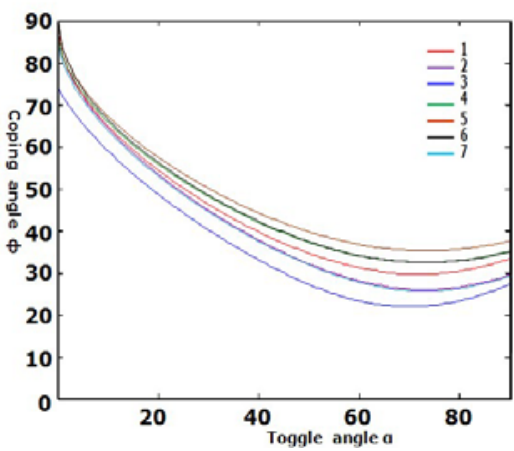

Fig.6 Relationship between coping angle and toggle angle of toggle mechanism

Analysis of data

The relationship between toggle angle and stroke ratio, velocity ratio, amplified force ratio and the coping angle based on MATLAB are showed from Fig.3 to Fig.6. From the figures, the change of parameters in different designed parameters can be described as follows:

(1) From Fig.3, the stroke ratio of third group and sixth group is higher than that of first group. And the other groups under the first group can be eliminated because of no improvement. As can be seen from fig.4, the velocity ratio of the third and sixth group are better than that of the first group, and the sixth group has the better curve because it's velocity increases faster than other groups.

(2) In Fig.5, it can be seen that the change of toggle angle has little effect on the amplified force according to the superposition of most of the curves. It is obvious that the third group has bad effects on the increase of the amplified force in the clamping mechanism, so it can be ignored. Fig.6 shows that the curves of the fourth, fifth and sixth group are located above the curve of the first group. Because the entire minimal coping angle has an increasing trend, these three groups of data can be used for analysis.

Based on the above analysis, the sixth group data contributes to the improvement of all the parameter of double-toggle clamping mechanism. So it can be used as the optimized parameters of double-toggle clamping mechanism to guide more experiments on actual design and manufacture of clamping mechanism.

\section{Conclusions}

(1) This paper is based on the geometric model of double-toggle clamping mechanism. After analyzing the mechanical and kinematic performance of the mechanism specially, the mathematic expression of geometry and position dimensions, stroke, velocity and force amplified ratio, which settles theoretic foundation for the calculation analysis of MATLAB.

(2) In order to obtain the curves of the parameters expediently, the mechanical and kinematic performance of the double-toggle clamping mechanism is analyzed by MATLAB in this paper. Then the clamping mechanism is designed with better parameters, which avoid repeated experiments under different parameters. The analysis of MATLAB can improve the design efficiency.

\section{Acknowledgement}

This study was funded by the National Natural Science Foundation of China (No.51173015, No.51203010)

\section{Reference}

[1] Hongtao Ni. Research of the optimal model of clamping system for injection molding machine [J].Light Industry Machinery, 2007(25):33-35.

[2] MingShan Huang, TsungYen Lin, Rong-Fong Fung. Key design parameters and optimal design 
of a five-point double-toggle clamping mechanism[J].Applied Mathematical Modeling, 2011(35): $4304-4320$.

[3] Chenshi Zhu, Qiong Wu, Tiejun Li, Hongqi Ni, Xin Li, Tiexin Fang. Establishment of motional and mechanical model for oblique five-hinge joint double toggle clamping unit of injection molding machine[J].Journal of shenyang institute of chemical technology,2004(18):133-137.

[4] Hui Yin, Guodong Lu, Jin Wang, Songhua Wang. Analysis and development mechanisms of injection trend of clamping molding machines [J].China Plastics, 2009(23):1-6.

[5] Guobao Wang, Hang Cheng, Fu Li,Liang Lu, Yali Ma. Research on optimization design of double toggle clamping unit for injection molding mechanism $[\mathrm{J}]$. Engineering plastics applications, 2011(39):87-90.

[6] Xingtian Wang. Injection molding process and equipment [M].Chemical Industry Press, 2010:580-592.

[7] Beijing Institute of Chemical Technology, South China Institute of Technology. Mechanical Design and Theory[M].Light Industry Press,1983: 434-448. 